\title{
The feasibility and advantage of uniportal video-assisted thoracoscopic surgery (VATS) in pulmonary lobectomy
}

\author{
Linlin Wang ${ }^{1,2}$, Dabei Liu, ${ }^{1,2}$ Jibin Lu², Suning Zhang ${ }^{2}$ and Xueying Yang ${ }^{1 *}$
}

\begin{abstract}
Background: Ongoing improvements in technique and instruments for video-assisted thoracoscopic surgery (VATS) have made minimally-invasive uniportal VATS lobectomy a reality. However, the outcomes of the procedure are still under investigation, and at present, uniportal VATS lobectomy is performed infrequently at most hospitals. We have therefore reviewed our outcomes with this procedure in an attempt to validate its safety, efficacy, and feasibility.

Methods: We retrospectively analyzed and compared perioperative data for patients who underwent uniportal, two-port, and traditional three-port VATS lobectomy between January 2015 and December 2015 at our hospital.

Results: Among 257 patients who had successful VATS lobectomy during the study period, 73 underwent uniportal VATS, 86 underwent two-port VATS, and 98 underwent traditional three-port VATS. There were no surgical or 30-day postoperative mortalities, and no significant differences in operative times, blood loss, number of lymph nodes retrieved and nodal stations explored, drainage times, length of hospital stay, or postoperative complications among the three groups. The visual analogue scale (VAS) pain scores were significantly lower in the uniportal VATS group after surgery $(P<0.05)$.

Conclusions: Uniportal VATS lobectomy is a safe and feasible surgical procedure that is associated with decreased surgical trauma and less postoperative pain compared to traditional VATS. Further long term follow-up analyses in large numbers of patients are ongoing.
\end{abstract}

Keywords: Uniportal, VATS, Lobectomy, Minimally invasive surgery

\section{Background}

Rapid technological progress has allowed thoracic surgery to become more minimally invasive with faster postoperative rehabilitation [1]. Conventional threeport video-assisted thoracic surgery (VATS) lobectomy is well established, and many units have successfully adopted this technique over the past two decades [2]. Since 2010, uniportal VATS lobectomy has become a new area of exploration in minimally invasive thoracic surgery [3]. Uniportal VATS is a less invasive approach that allows major thoracic operations to be performed through a single small incision of about $4 \mathrm{~cm}$. With consistent reports of excellent results, uniportal VATS

\footnotetext{
* Correspondence: yangxy@sj-hospital.org

${ }^{1}$ Department of Thoracic Surgery, The Fourth Affiliated Hospital of China Medical University, No. 4, CongShan East Road, HuangGu District, Shenyang 110032, Liaoning, People's Republic of China

Full list of author information is available at the end of the article
}

is becoming more and more widely accepted $[4,5]$. The advantages of uniportal VATS can include reduced surgical trauma, decreased postoperative pain, faster rehabilitation, and improved patient satisfaction with a less invasive approach than conventional VATS $[6,7]$. However, concerns about operative risks, technical challenges, and unstudied outcomes of uniportal VATS lobectomy remain. Therefore, the aim of this study is to assess the feasibility and applicability of the uniportal VATS approach in pulmonary lobectomy, and to compare perioperative outcomes of uniportal VATS with those of two-port and traditional three-port VATS.

\section{Methods}

\section{Data acquisition and follow-up}

We retrospectively analyzed results in patients who underwent uniportal, two-port, or traditional three-port 
VATS lobectomy between January 2015 and December 2015 at our hospital. None of the patients had received neoadjuvant therapy before surgery. Institutional review board (IRB) approval and written informed consent were obtained. Patients underwent routine preoperative arterial blood gas analysis, pulmonary function testing, bronchoscopy, and computed tomography. Pre-, peri-, and postoperative patient details and outcome variables were collected by means of clinical assessment, patient inquiry, and review of any inpatient admissions.

\section{Surgical technique}

Until 2014 we employed only the traditional three-port VATS technique for pulmonary lobectomy, involving 3 incisions with the operative field visualized on screen via video thoracoscope [8]. In 2014, we began performing two-port VATS lobectomy. In the two-port procedure, the video thoracoscope is introduced through the 7th or 8th intercostal space in the midaxillary line, and the operation is performed through an incision of approximately $4 \mathrm{~cm}$ that is placed anteriorly in the 4th or 5th intercostal space with no rib spreading. This incision serves as the utility port for the passage of instruments and staplers and ultimately for extraction of the specimen. Our substantial experience with conventional and double-port VATS prepared us to begin performing uniportal VATS lobectomies with no rib spreading beginning in January 2015.
Uniportal video assisted thoracoscopic surgery technique Uniportal VATS was performed with patients under general anesthesia with single-lung ventilation via a double lumen endotracheal tube. The patients were placed in a full lateral decubitus position with the operating table flexed to increase the intercostal spacing. A single incision of approximately $4 \mathrm{~cm}$ was made in the 4 th or 5 th intercostal space at the anterior axillary line to facilitate good access to hilar structures and lymph node stations (Fig. 1a). Both the surgeon and the assistant stood at the anterior side of the patient so that they had the same field of vision and were better coordinated. We placed a special plastic wound protector (Demai, China) in the incision to form two channels, one for the thoracoscope and the other for the surgical instruments (Fig. 1a). The thoracoscope was usually placed through the upper channel with the instruments in the lower channel, but the scope and the instruments could be easily transposed as needed. We used Ethicon (Ethicon Endo-Surgery, LLC, USA) brand instruments (i.e. Echelon Flex 45 articulating endoscopic linear cutters), as well as long curved endoscopic instruments with double articulations, curved suction (Yundi, China), and a $30^{\circ}$ thoracoscope.

The surgeon performed the operation by bimanual instrumentation with a pair of surgical instruments that was crossed at the incision site (Fig. 1a). The surgical steps were similar to those carried out during traditional VATS, including individual dissection of the veins, arteries, and lobar
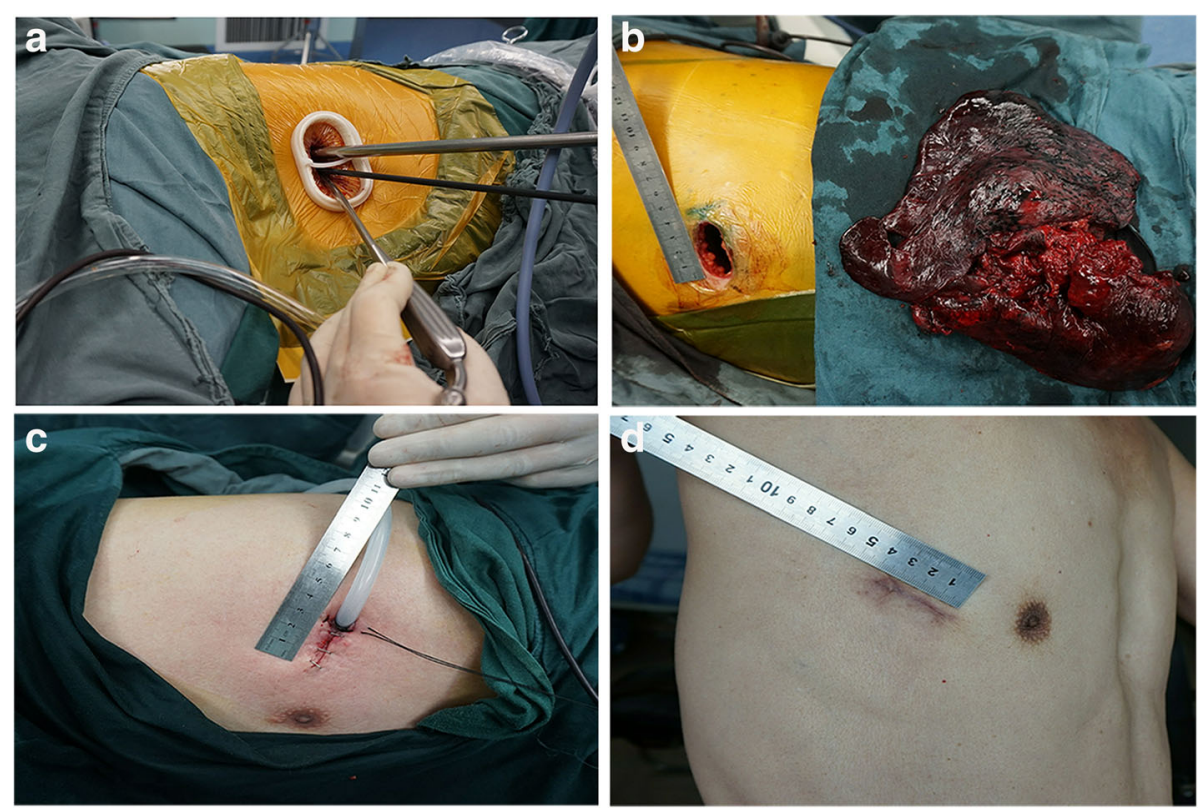

Fig. 1 Uniportal video assisted thoracoscopic surgery (VATS) lobectomy. A single incision of approximately $4.0 \mathrm{~cm}$ is made in the 4th or 5 th intercostal space at the anterior axillary line. a A specially-designed wound protector is inserted, forming two channels, one for the thoracoscope and the other for the surgical instruments. b Left pneumonectomy. c A U-shaped suture is placed around the chest tube in order to close the incision immediately after removal of the tube. $\mathbf{d}$ One month after right upper lobectomy 
bronchus and complete mediastinal lymphadenectomy, and the instrumentation for obtaining the target tissue in the single-port approach mimicked that of conventional thoracotomy [9]. We generally followed an operative sequence of vein, artery, and bronchus. However, for the left upper lobe we preferred the sequence of artery, vein, and bronchus because of the anatomical features. We used a Flex 45 endostapler (Ethicon) to manage the bronchus, incomplete fissures, and the main blood vessel, while smaller vascular branches were best addressed by silk ligature and vascular clips. Once separated, the resected lobe was placed in a protective bag and removed through the single incision (Fig. 1b). Complete mediastinal lymphadenectomy was performed in lung cancer patients, and the number of accessible lymph node stations was equivalent to that of open thoracotomy. At the end of the operation, we secured the chest tube with a U-shaped suture to allow immediate closure of the wound upon removal of the tube (Fig. 1c), and the wound was then infiltrated with ropivacaine (Naropin, AstraZeneca AB, Sweden). Lesions were localized preoperatively in patients with pulmonary ground-glass opacity (GGO) and those with small nodules by using a CT-guided puncture positioning method.

\section{Visual analog scale (VAS) pain scores}

The intensity of postoperative pain was scored with a 10-cm vertical visual analogue scale (VAS) [10] anchored at the bottom by "no pain" and at the top by "worst imaginable pain" and marked at predetermined intervals. The patients rated their pain by marking the appropriate point between the two extremes, and the scores were determined by measuring the distance from the minimal endpoint to the mark. Pain scores were assessed at 24, 48 , and $72 \mathrm{~h}$ postoperatively and at 1 week and 1 month after surgery.

\section{Statistical analysis}

Categorical variables were expressed as percentages and continuous variables were expressed as mean \pm standard deviation. Variables were compared using Student's $t$ test, the $\chi 2$ test and variance analysis or Fisher's exact test. Data were analyzed with SPSS 17.0 software. Pvalues $<0.05$ were considered statistically significant.

\section{Results}

\section{Patient characteristics}

There were 257 patients who underwent successful VATS lobectomy from January 2015 to December 2015, and there were no intraoperative or 30-day mortalities. Seventy-three patients underwent uniportal VATS, 86 underwent two-port VATS, and 98 underwent traditional three-port VATS. All types and combinations of lobectomy were performed (Table 1). Postoperative pathologic diagnoses included both primary lung cancers and benign conditions (Table 2). Primary lung cancers were classified by pathologic stage (stage I, stage II, or stage III or higher; Table 3).

\section{Surgical outcomes}

There were 1, 2, and 2 procedures converted to conventional open thoracotomy in the uniportal, two-port, and three-port VATS groups, respectively, for conversion rates of $1.37 \%, 2.33 \%$, and $2.04 \%(P>0.05)$. Conversions were mainly due to bleeding and dense adhesions. All conversions were completed safely and no inpatient deaths occurred. There were no significant differences in operative times, blood loss, number of lymph nodes retrieved and nodal stations explored, drainage times, or length of hospital stay among the three groups (Table 4). There was no intraoperative mortality in any of the three groups. The perioperative (30-day) mortality was $0 \%$ in the uniportal and three-port VATS groups and $1.16 \%$ in the two-port VATS group, where one patient died of respiratory failure. Four patients $(5.48 \%), 9$ patients $(10.47 \%)$, and 9 patients $(9.18 \%)$ had postoperative complications $(P>0.05)$ (Table 5).

\section{Visual analogue scale pain scores}

In the uniportal VATS group, the mean VAS scores at 24,48 , and $72 \mathrm{~h}$ and 1 week and 1 month after surgery were $5.51 \pm 1.62,4.17 \pm 1.44,3.21 \pm 1.32,1.83 \pm 0.47$, and $0.79 \pm 0.49$. The VAS scores in the two-port VATS group were $7.67 \pm 0.82,6.00 \pm 0.63,4.17 \pm 0.75,2.17 \pm 0.41$, and $0.83 \pm 0.41$, and in the three-port VATS group, the scores were $7.88 \pm 1.20,6.24 \pm 1.24,4.52 \pm 1.26,2.20 \pm$ 0.50 , and $1.08 \pm 0.40$. The postoperative pain scores were significantly lower $(P<0.05)$ in the uniportal VATS group compared to the other two groups, confirming an advantage for uniportal VATS in terms of reduced pain during the early postoperative period (Fig. 2).

\section{Discussion}

The surgical approach to lung resection is constantly evolving [11]. Experience gained through routine application of traditional VATS techniques combined with ongoing improvements in the surgical instruments have led to great advances in minimally invasive thoracic surgery [12]. The first uniportal VATS lobectomy was reported by Gonzalez-Rivas et al. in 2011 [3], and the technique and reliability of this approach have been improving steadily ever since [13]. Uniportal VATS has changed our outlook on minimally invasive thoracic surgery. Although it is technically demanding, once mastered, uniportal VATS can be expected to minimize the amount of surgical trauma, the advantages of which include decreased postoperative pain, shorter hospital stay, and preservation of pulmonary function, promoting more rapid recovery and providing superior cosmetic 
Table 1 Baseline patient characteristics

\begin{tabular}{|c|c|c|c|c|}
\hline Characteristic & Uniportal VATS $(n=73)$ & Two-port VATS $(n=86)$ & Three-port VATS $(n=98)$ & $P$-value \\
\hline Age (Mean \pm SD) & $57.12 \pm 6.43$ & $54.36 \pm 7.6$ & $61.32 \pm 7.54$ & $<0.001$ \\
\hline Sex (\%) & & & & 0.288 \\
\hline Male & $31(42.47 \%)$ & $45(52.33 \%)$ & $53(54.08 \%)$ & \\
\hline Female & $42(51.53 \%)$ & $41(47.67 \%)$ & $45(45.92 \%)$ & \\
\hline \multicolumn{5}{|c|}{ Pulmonary function (Mean \pm SD) } \\
\hline FVC (\% predicted) & $90.83 \pm 13.43$ & $88.32 \pm 15.87$ & $85.33 \pm 15.87$ & 0.064 \\
\hline FEV1 (\% predicted) & $86.18 \pm 12.91$ & $82.70 \pm 16.30$ & $80.71 \pm 19.08$ & 0.104 \\
\hline MW (\% predicted) & $69.26 \pm 18.84$ & $67.75 \pm 25.10$ & $70.78 \pm 21.43$ & 0.649 \\
\hline \multicolumn{5}{|c|}{ Arterial blood gas analysis (Mean \pm SD) } \\
\hline $\mathrm{PAO}_{2}(\mathrm{mmHg})$ & $84.87 \pm 12.62$ & $83.10 \pm 18.43$ & $79.75 \pm 14.20$ & 0.085 \\
\hline $\mathrm{PACO}_{2}(\mathrm{mmHg})$ & $41.26 \pm 5.01$ & $42.37 \pm 5.85$ & $42.61 \pm 6.12$ & 0.286 \\
\hline $\mathrm{SAO}_{2}(\%)$ & $96.55 \pm 1.42$ & $95.58 \pm 2.47$ & $95.72 \pm 3.37$ & 0.048 \\
\hline \multicolumn{5}{|l|}{ Lobectomy (\%) } \\
\hline Right upper & $16(21.92 \%)$ & $28(32.56 \%)$ & $23(23.47 \%)$ & 0.237 \\
\hline Right middle & $4(5.48 \%)$ & $6(6.98 \%)$ & $11(11.22 \%)$ & 0.352 \\
\hline Right lower & $18(24.66 \%)$ & $13(15.12 \%)$ & $23(23.47 \%)$ & 0.254 \\
\hline Right middle and lower & $3(4.11 \%)$ & $1(1.16 \%)$ & $0(\%)$ & 0.069 \\
\hline Left upper & $12(16.44 \%)$ & $16(18.6 \%)$ & $24(24.49 \%)$ & 0.388 \\
\hline Left lower & $19(26.03 \%)$ & $22(25.58 \%)$ & $16(16.33 \%)$ & 0.207 \\
\hline Left pneumonectomy & $1(1.37 \%)$ & $0(\%)$ & $1(1.02 \%)$ & 0.744 \\
\hline
\end{tabular}

FVC forced vital capacity, FEV1 forced expiratory volume in 1 second, $M V V$ maximal ventilatory volume, $P_{2} O_{2}$ partial pressure of oxygen in artery, $P A C O_{2}$ partial pressure of carbon dioxide in artery, $\mathrm{SAO}_{2}$ arterial oxygen saturation

Table 2 Pathology results

\begin{tabular}{|c|c|c|c|c|}
\hline Pathological types & Uniportal VATS (\%) $(n=73)$ & Two-port VATS (\%) $(n=86)$ & Three-port VATS $(\%)(n=98)$ & $P$-value \\
\hline Primary lung cancer & $51(69.86 \%)$ & $56(65.12 \%)$ & $71(72.45 \%)$ & 0.556 \\
\hline Adenocarcinoma & $34(46.58 \%)$ & $38(44.19 \%)$ & $52(53.06 \%)$ & 0.459 \\
\hline Squamous cell carcinoma & $16(21.92 \%)$ & $15(17.44 \%)$ & $13(13.27 \%)$ & 0.330 \\
\hline Adenosquamous & $0(0 \%)$ & $1(1.16 \%)$ & $1(1.02 \%)$ & 1.000 \\
\hline Large cell carcinoma & $1(1.37 \%)$ & $0(0 \%)$ & $2(2.04 \%)$ & 0.506 \\
\hline Carcinoid tumors & $0(0 \%)$ & $1(1.16 \%)$ & $0(0 \%)$ & 0.619 \\
\hline Carcinosarcoma & $0(0 \%)$ & $0(0 \%)$ & $1(1.02 \%)$ & 1.000 \\
\hline Small cell carcinoma & $0(0 \%)$ & $1(1.16 \%)$ & $2(2.04 \%)$ & 0.780 \\
\hline Benign disease & $22(30.14 \%)$ & $30(34.88 \%)$ & $27(27.55 \%)$ & 0.556 \\
\hline Inflammation & $3(4.11 \%)$ & $6(6.98 \%)$ & $7(7.14 \%)$ & 0.676 \\
\hline Pulmonary cyst & $4(5.48 \%)$ & $4(4.65 \%)$ & $4(4.08 \%)$ & 0.933 \\
\hline Tuberculosis & $4(5.48 \%)$ & $9(10.47 \%)$ & $7(7.14 \%)$ & 0.482 \\
\hline Pulmonary hamartoma & $2(2.74 \%)$ & $3(3.49 \%)$ & $3(3.06 \%)$ & 1.000 \\
\hline Pulmonary fibrosis & $4(5.48 \%)$ & $3(3.49 \%)$ & $1(1.02 \%)$ & 0.223 \\
\hline Bronchiectasis & $5(6.85 \%)$ & $4(4.65 \%)$ & $4(4.08 \%)$ & 0.723 \\
\hline Lung sequestration & $0(0 \%)$ & $0(0 \%)$ & $1(1.02 \%)$ & 1.000 \\
\hline Spindle cell lipoma & $0(0 \%)$ & $1(1.16 \%)$ & $0(0 \%)$ & 0.619 \\
\hline
\end{tabular}

There were no significant difference in these results among the three groups (all $P>0.05$ ) 
Table 3 Distribution of primary lung cancers by pathologic stage

\begin{tabular}{|c|c|c|c|c|}
\hline Stage & Uniportal VATS $(\%)(n=51)$ & Two-port VATS (\%) $(n=56)$ & Three-port VATS $(\%)(n=71)$ & $P$-value \\
\hline Stage I & 27 (52.94\%) & $29(51.79 \%)$ & $34(47.89 \%)$ & 0.839 \\
\hline IA & $13(25.49 \%)$ & $13(23.21 \%)$ & $19(26.76 \%)$ & 0.900 \\
\hline IB & $14(27.45 \%)$ & $16(28.57 \%)$ & $15(21.13 \%)$ & 0.578 \\
\hline Stage II & $21(41.18 \%)$ & $24(42.86 \%)$ & $30(42.25 \%)$ & 0.984 \\
\hline$\| \mathrm{A}$ & $11(21.57 \%)$ & $14(25.00 \%)$ & $14(19.72 \%)$ & 0.773 \\
\hline$\| \mathrm{B}$ & $10(19.61 \%)$ & $10(17.86 \%)$ & $16(22.54 \%)$ & 0.802 \\
\hline Stage III or greater & $3(5.88 \%)$ & $3(5.36 \%)$ & $7(9.86 \%)$ & 0.625 \\
\hline$\| \mathrm{A}$ & $3(5.88 \%)$ & $3(5.36 \%)$ & $5(7.04 \%)$ & 0.928 \\
\hline$\| \mathrm{IIB}$ & $0(0 \%)$ & $0(0 \%)$ & $1(1.41 \%)$ & 1.000 \\
\hline $\mathrm{IV}^{\mathrm{a}}$ & $0(0 \%)$ & $0(0 \%)$ & $1(1.41 \%)$ & 1.000 \\
\hline
\end{tabular}

${ }^{a}$ Isolated brain metastasis following metastasectomy prior to being evaluated for lobectomy

There were no significant difference in these results among the three groups (all $P>0.05$ )

results when compared with pulmonary lobectomy via traditional three-port VATS $[14,15]$. Uniportal VATS is also advantageous to the surgeon because it provides a more anatomic and direct view of the target tissues and it allows bimanual instrumentation, as in an open approach. The uniportal approach also negates the creation of torsional or dihedral angles by the instruments that is inherent to conventional multiport VATS [16].

Following the advances in video endoscopic instruments and endosurgical techniques, the indications for uniportal VATS lobectomy have been expanded to a larger patient population, and it is now a feasible option for treatment of a variety of benign lung diseases as well as for lung cancers that are amenable to complete resection by lobectomy. Greater numbers of surgeons are now gaining the experience required to perform technically demanding uniportal VATS procedures via a small single incision with excellent postoperative results [17]. Prior thoracic irradiation and induction therapy, sleeve lobectomy, and vascular reconstruction need not be considered contraindications to uniportal VATS [18], and preoperative assessment and patient selection for uniportal VATS lobectomy should be conducted as for conventional three-port VATS [19]. We are now performing almost all of our VATS lobectomies, as well as pneumonectomies, via a uniportal approach.
Uniportal VATS has been reliably comparable to multiportal VATS in terms of safety and efficacy, but the geometric configuration of the approach is completely different. Uniportal VATS is geometrically favorable to multiport techniques. It provides direct in-plane visualization of the target tissues because the thoracoscope and the surgical instruments enter the chest through the same incision. Because uniportal VATS provides the surgeon with the perspective of operating in the same projection plane and retains visual depth, it is easier to judge distances and improve the accuracy of the surgical maneuvers. However, there are also some difficulties associated with the singleincision technique. The operating instruments are more prone to impeding one another's movements, and the assistant who is managing the thoracoscope may be more prone to fatigue and error. As mentioned, one of our techniques is to place a special plastic wound protector in the incision to form two separate channels, one for the thoracoscope and the other for the surgical instruments. This makes it possible to fix the thoracoscope in place and reduces the tendency to mutual interference of the instruments. The thoracoscope and the instruments, including the articulating endostapler and the long curved double articulation instruments, can easily be transposed for optimal positioning and angle of operation during surgery, and the demand on the assistant is lessened while the accuracy of

Table 4 Surgical results (Mean \pm SD)

\begin{tabular}{|c|c|c|c|c|}
\hline Characteristic & Uniportal VATS $(n=73)$ & Two-port VATS $(n=86)$ & Three-port VATS $(n=98)$ & $P$-value \\
\hline Operative time (min) & $154.88 \pm 31.31$ & $163.91 \pm 49.72$ & $162.84 \pm 68.18$ & 0.519 \\
\hline Blood loss (mL) & $92.5 \pm 22.66$ & $100 \pm 33.89$ & $103.21 \pm 27.41$ & 0.051 \\
\hline Drainage time (days) & $4.55 \pm 1.41$ & $5.34 \pm 1.81$ & $5.26 \pm 3.15$ & 0.069 \\
\hline Length of hospital stay (days) & $8.63 \pm 2.06$ & $8.95 \pm 2.40$ & $9.55 \pm 3.18$ & 0.069 \\
\hline Number of lymph nodes retrieved (lung cancer patients only) & $13.56 \pm 3.79$ & $12.68 \pm 3.17$ & $12.71 \pm 4.18$ & 0.252 \\
\hline Number of nodal stations explored (lung cancer patients only) & $7.05 \pm 1.15$ & $6.73 \pm 1.28$ & $6.56 \pm 1.49$ & 0.059 \\
\hline
\end{tabular}

There were no significant difference in these results among the three groups (all $P>0.05$ ) 
Table 5 Postoperative complications

\begin{tabular}{lllll}
\hline Complication & Uniportal VATS $(\%)(n=73)$ & Two-port VATS $(\%)(n=86)$ & Three-port VATS $(\%)(n=98)$ & 3 -value \\
\hline Pneumonia & $2(2.74 \%)$ & $3(3.49 \%)$ & $3.06 \%)$ & $3(3.06 \%)$ \\
Prolonged air leak & $1(1.37 \%)$ & $2(2.33 \%)$ & $1(1.02 \%)$ & 0.879 \\
Cardiac arrhythmias & $1(1.37 \%)$ & $2(2.33 \%)$ & $2(2.04 \%)$ & 0.832 \\
Hemothorax & $0(0 \%)$ & $1(1.16 \%)$ & $0(0 \%)$ & 0.780 \\
Respiratory failure & $0(0 \%)$ & $1(1.16 \%)$ & $9(9.18 \%)$ & 0.619 \\
Totals & $4(5.48 \%)$ & $9(10.47 \%)$ & 0.514
\end{tabular}

The overall rate of postoperative complications was no significantly differences (all $P>0.05$ ) in the uniportal VATS group compared to the two- and three-port VATS groups

the operation is improved. Another important issue during uniportal VATS approach is the difficulty in palpating small lung lesions through the single incision. We use a preoperative CT-guided puncture positioning method to localize small nodules and to pinpoint lesions in patients with pulmonary GGO. In addition, there has been some concern that placing the chest tube in the single incision could affect wound healing. We have addressed this by placing a Ushaped suture around the chest tube, which allows immediate closure of the wound upon removal of the tube, and have had excellent results (Fig. $1 \mathrm{c}, \mathrm{d}$ ).

In this study, we compared the outcomes of pulmonary lobectomy by uniportal VATS with those of lobectomy by two- and three-port VATS and found that there were no significant differences in operative time, blood loss, number of lymph nodes retrieved and nodal stations explored, drainage time, length of hospital stay, or postoperative complications among the three groups. Rates of conversion from VATS to open surgery ranging from 2 to $23 \%$ have been reported [20]. In our study, the rates of conversion were $1.37 \%, 2.33 \%$, and $2.04 \%$ for uniportal, two-port, and three-port VATS. Because discharge of our patients is delayed according to our customs, the median hospitalization period was longer compared to other hospitals, but there was no difference in length of stay among our three study groups. The average number of resected lymph nodes in our patients was lower than that in other reports [17] because we routinely perform en bloc resection of lymph nodes during mediastinal lymphadenectomy, and the number of lymph nodes retrieved and nodal stations explored did not differ among our three study groups. Finally, we found that the VAS pain scores were significantly lower after surgery in uniportal VATS group compared to the other two groups. We attributed this to reduced access trauma and reduced risk of intercostal nerve injury.

\section{Conclusion}

Uniportal VATS lobectomy is a safe and feasible surgical procedure that is associated with reduced surgical trauma and decreased postoperative pain compared to traditional VATS. However, uniportal VATS lobectomy

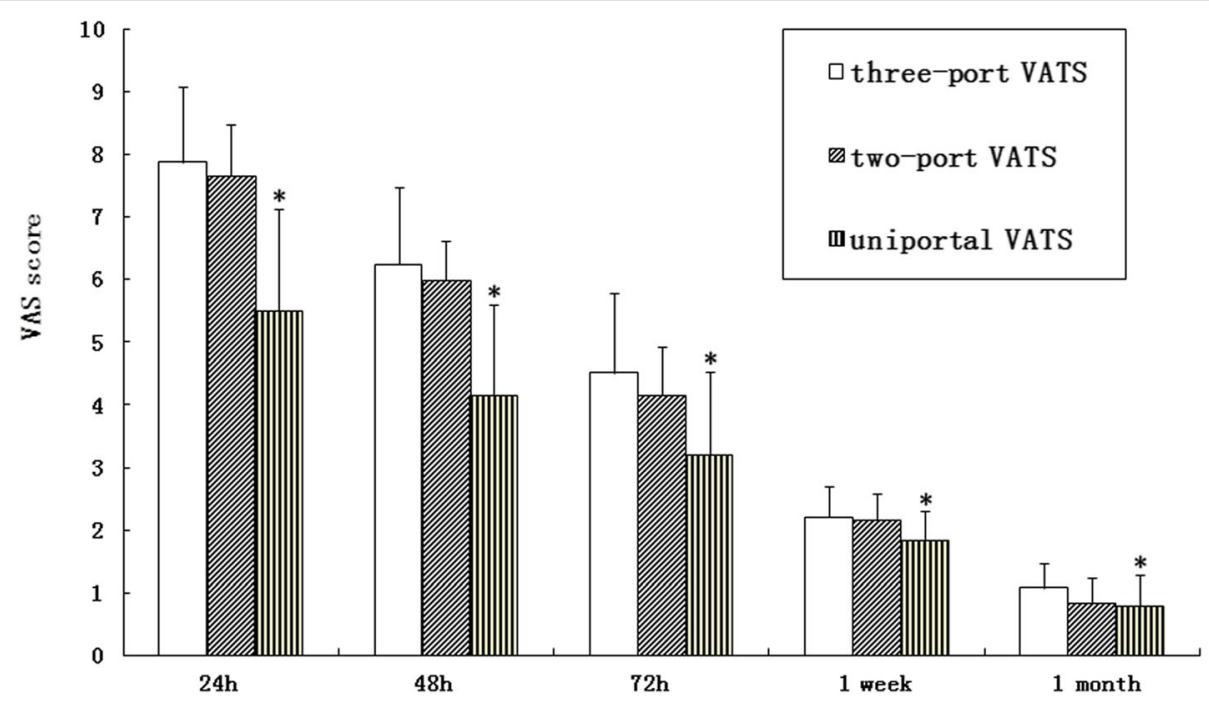

Fig. 2 Visual analog scale (VAS) pain scores. The postoperative pain scores in the uniportal VATS group after surgery were significantly lower compared to those of the two-port and three-port VATS groups $(P<0.05)$ 
is a relatively complex procedure and the experience of the surgeon along with the correct instrumentation are critical to its success. Rigorous training is required for this technique, and additional long-term survival and outcomes analyses should be conducted on larger numbers of patients. We can then anticipate that uniportal VATS lobectomy will be more and more widely performed in the years to come.

\section{Abbreviations}

CT: Computed tomography; GGO: Ground-glass opacity; IRB: Institutional review board; SD: Standard deviation; VAS: Visual analog scale; VATS: Videoassisted thoracoscopic surgery

\section{Acknowledgements}

Not applicable.

\section{Funding}

This study was supported by the Specialized Research Fund for the Doctoral Program of Higher Education of China (20122104120015). The funding agency has not played a role in the design of the study and collection, analysis, interpretation of data and writing the manuscript.

\section{Availability of data and materials}

The datasets supporting the conclusions of this article are included within the article.

\section{Authors' contributions}

LLW and XYY drafted the manuscript. The data acquisition was performed by LLW and DBL. JBL and SNZ designed the analysis. XYY participated in the conception and design. All authors read and approved the final manuscript.

\section{Competing interests}

The authors declare that they have no competing interests.

\section{Consent for publication}

Not applicable.

\section{Ethics approval and consent to participate}

This study was approved by the Ethics committee of Shengjing Hospital of China Medical University. Written informed consent was obtained from each patient in this study.

\section{Author details}

'Department of Thoracic Surgery, The Fourth Affiliated Hospital of China Medical University, No. 4, CongShan East Road, HuangGu District, Shenyang 110032, Liaoning, People's Republic of China. ${ }^{2}$ Department of Thoracic Surgery, Shengjing Hospital of China Medical University, No. 36 Sanhao St., HePing District, Shenyang 110004, Liaoning, People's Republic of China.

Received: 25 October 2016 Accepted: 18 January 2017

Published online: 25 January 2017

\section{References}

1. Hanna WC, De Valence M, Atenafu EG, Cypel M, Waddell TK, Yasufuku K, et al. Is video-assisted lobectomy for non-small-cell lung cancer oncologically equivalent to open lobectomy? Eur J Cardiothorac Surg. 2013:43:1121-5.

2. Yan TD, Cao C, D'Amico TA, Demmy TL, He J, Hansen H, et al. Video-assisted thoracoscopic surgery lobectomy at 20 years: a consensus statement. Eur J Cardiothorac Surg. 2014;45:633-9.

3. Gonzalez-Rivas D, de la Torre M, Fernandez R, Mosquera VX. Single-port video-assisted thoracoscopic left upper lobectomy. Interact Cardiovasc Thorac Surg. 2011;13:539-41.

4. Rocco G. One-port (uniportal) video-assisted thoracic surgical resections-a clear advance. J Thorac Cardiovasc Surg. 2012;144:S27-31.

5. Anile M, Diso D, De Giacomo T, Rendina EA, Venuta F. Uniportal thoracoscopic lobectomy. Ann Thorac Surg. 2013;96:745.

6. Mier JM, Chavarin A, Izquierdo-Vidal C, Fibla JJ, Molins L. A prospective study comparing three-port video-assisted thoracoscopy with the single- incision laparoscopic surgery (SILS) port and instruments for the video thoracoscopic approach: a pilot study. Surg Endosc. 2013;27:2557-60.

7. Rocco G, Martucci N, La Manna C, Jones DR, De Luca G, La Rocca A, et al. Ten-year experience on 644 patients undergoing single-port (uniportal) video-assisted thoracoscopic surgery. Ann Thorac Surg. 2013;96:434-8.

8. Yang X, Wang S, Qu J. Video-assisted thoracic surgery (VATS) compares favorably with thoracotomy for the treatment of lung cancer: a 5-year outcome comparison. World J Surg. 2009;33:1857-61.

9. Wang BY, Tu CC, Liu CY, Shih CS, Liu CC. Single-incision thoracoscopic lobectomy and segmentectomy with radical lymph node dissection. Ann Thorac Surg. 2013;96:977-82

10. DeLoach LJ, Higgins MS, Caplan AB, Stiff JL. The visual analog scale in the immediate postoperative period: intrasubject variability and correlation with a numeric scale. Anesth Analg. 1998;86:102-6.

11. Reck M, Heigener DF, Mok T, Soria JC, Rabe KF. Management of non-smallcell lung cancer: recent developments. Lancet. 2013;382:709-19.

12. Ng CS, Lau KK, Gonzalez-Rivas D, Rocco G. Evolution in surgical approach and techniques for lung cancer. Thorax. 2013;68:681.

13. Gonzalez-Rivas D, Paradela M, Fernandez R, Delgado M, Fieira E, Mendez L, et al. Uniportal video-assisted thoracoscopic lobectomy: 2 years of experience. Ann Thorac Surg. 2013;95:426-32.

14. Yang HC, Cho S, Jheon S. Single-incision thoracoscopic surgery for primary spontaneous pneumothorax using the SILS port compared with conventional three-port surgery. Surg Endosc. 2013;27:139-45.

15. Tam JK, Lim KS. Total muscle-sparing uniportal videoassisted thoracoscopic surgery lobectomy. Ann Thorac Surg. 2013:96:1982-7.

16. Bertolaccini L, Rocco G, Viti A, Terzi A. Geometrical characteristics of uniportal VATS. J Thorac Dis. 2013:5 Suppl 3:S214-6.

17. Hsu PK, Lin WC, Chang YC, Chan ML, Wang BY, Liu CY, et al. Multiinstitutional Analysis of Single-Port Video-Assisted Thoracoscopic Anatomical Resection for Primary Lung Cancer. Ann Thorac Surg. 2015;99:1739-44.

18. Gonzalez-Rivas D, Fernandez R, Fieira E, Rellan L. Uniportal video-assisted thoracoscopic bronchial sleeve lobectomy: first report. J Thorac Cardiovasc Surg. 2013;145:1676-7.

19. Ibrahim M, Menna C, Andreetti C, D'Andrilli A, Ciccone AM, Maurizi G, et al. Flexible videoscope for thoracoscopic lobectomy: evolution of uniportal technique. Surg Endosc. 2015:29:2056-9.

20. Mahtabifard A, DeArmond DT, Fuller CB, McKenna Jr RJ. Video-assisted thoracoscopic surgery lobectomy for stage I lung cancer. Thorac Surg Clin. 2007:17:223-31.

Submit your next manuscript to BioMed Central and we will help you at every step:

- We accept pre-submission inquiries

- Our selector tool helps you to find the most relevant journal

- We provide round the clock customer support

- Convenient online submission

- Thorough peer review

- Inclusion in PubMed and all major indexing services

- Maximum visibility for your research

Submit your manuscript at www.biomedcentral.com/submit
Biomed Central 